\title{
Nuevas TIC en los Andes peruanos: fundamentos teóricos y balance bibliográfico
}

\author{
Mario Sánchez Dávila \\ Pontificia Universidad Católica del Perú (PUCP) \\ Universidad Peruana de Ciencias Aplicadas (UPC)
}

Recibido: $18 / 10 / 2016$

Aprobado: 2/12/2016

\begin{abstract}
RESUMEN. En primer lugar, este artículo explica los fundamentos teóricos en los cuales se sustenta la presente propuesta de antropología digital en los Andes peruanos (desde los orígenes de la antropología digital, los debates sobre la tecnología oral y escrita, y las teorías sobre la tecnología digital como práctica social). En segundo lugar, este artículo realiza un balance bibliográfico de los estudios sobre las nuevas TIC en los Andes peruanos (desde la expresión identitaria, el desarrollo productivo y la educación formal en el mundo andino).

Palabras clave: tecnología / antropología / digital / Andes / Perú
\end{abstract}

\section{New ICT in the Peruvian Andes: Theoretical Foundation and Bibliographical Balance}

Авsтract. On the one hand, this paper explains the theoretical foundations on which this proposal for digital anthropology in the Peruvian Andes is based (on the origins of digital anthropology, discussions on oral and written technology, and theories of digital technology as social practice). And, on the other hand, this paper presents a bibliographical balance of the studies on the new ICT in the Peruvian Andes (on identity expression, productive development and formal education in the Andean world).

Keywords: technology / Anthropology / digital / Andes / Peru 
E ste artículo explora teorías y estudios para una propuesta de antropología digital en los Andes peruanos. Por un lado, se explican los fundamentos teóricos en los cuales se sustenta una antropología digital en los Andes peruanos, enfatizando a) Los orígenes de la antropología digital (desde una economía política de los medios de comunicación masiva y un enfoque poscolonial de las nuevas TIC); y b) La tecnología digital como práctica social (desde los discursos como microrrepresentaciones ideológicas y relaciones de poder; los usos y sus condiciones de domesticación productiva, económica y técnica; el rol de los contextos, actores, propósitos y resultados). Por otro lado, se realiza un balance bibliográfico de los estudios sobre las nuevas TIC en los Andes peruanos (desde la expresión identitaria, el desarrollo productivo y la educación formal en el mundo andino).

\section{Tradición y modernidad en los Andes: una introducción}

La tecnología en el mundo andino no constituye, en sí misma, una novedad, pues las culturas andinas han tenido presencia y permanencia de tecnologías dentro de su vida social antes y después de la conquista hispana: tecnología medicinal (hecha de minerales, vegetales y productos biológicos animales y humanos), tecnología musical (el clarín, el pincullo, la tinya y el waqrapuku), tecnología climática (conocimientos de los fenómenos meteorológicos para pronósticos del tiempo), tecnología de registro y contabilidad (los quipus), tecnología de conservación alimentaria (las colcas), tecnología ganadera (bofedales y pastizales; tratamientos de fracturas en ovinos; dosificaciones contra enfermedades hepáticas y gastropulmonares del ganado) y tecnología agrícola (los sistemas de andenería, riego y camellones; técnicas usadas en el proceso de labranza, preparación del terreno, abonamiento y siembra, controles fitosanitarios, cosecha y almacenamiento; herramientas empleadas en estos procesos, como la chaquitaclla y el arado; y el conocimiento de plantas como indicadoras de la calidad del suelo), entre otras manifestaciones tecnológicas en el mundo andino (véase otros ejemplos de tecnologías andinas en Bustamante, 1988; el Proyecto de tecnologías campesinas del CEDECUM, 1988; el tomo VI del Festival del libro puneño II, 1987; en Rengifo et al., 1986; y Lechtman y Soldi, 1981). Es por eso que el interés de este artículo no radica en las tecnologías en el mundo andino, sino en las nuevas tecnologías de información y comunicación (nuevas TIC) en los Andes peruanos.

Si los estudios etnohistóricos, basados en la documentación de los visitadores y cronistas españoles, arrojaron valiosos alcances para la comprensión social y cultural de los Andes prehispánicos y coloniales, los estudios antropológicos produjeron sustanciales hallazgos para la comprensión del 
mundo andino republicano y contemporáneo, diagnosticando, a través de la investigación etnográfica, las estructuras sociales de la organización familiar, comunal, agrícola, ganadera, económica, política, religiosa, ritual y mitológica, entre otras formas de ser-hacer-estar, en la cultura andina peruana. Por ello, las investigaciones de antropología digital en los Andes peruanos complementarían los estudios de antropología andina, examinando las múltiples y complejas formas en que las estructuras sociales del mundo andino son mediadas por las nuevas TIC y sus prácticas sociales (discursos y usos), poniendo en evidencia cómo los escenarios rurales de las comunidades, en el siglo xxI, continúan preservando la tradición de sus herencias culturales mientras se encuentran insertos en modernos cambios sociales que conllevan los dinámicos y constantes procesos de interrelación con sociedades urbanas y globales capitalistas.

$\mathrm{Y}$ es que, como bien es sabido, el mundo andino peruano ha pasado por una amplia gama de procesos de interrelación sociocultural (presentes en las conquistas lingüístico-políticas, en los préstamos religioso-mitológicos o en los intercambios agropecuarios), que acontecieron mucho antes del advenimiento de las nuevas TIC del siglo xxI y de los medios de comunicación masiva del siglo xx, de la revolución industrial del siglo XVIII, de la conquista española del siglo xvI, e incluso del encuentro oficial del "viejo" y el "nuevo" mundo del siglo $\mathrm{xv}$.
Es precisamente por ello que, hablando epistemológicamente, es necesario conceptualizar la tradición y la modernidad en los Andes peruanos como dos categorías complementarias e interrelacionadas, y no como mutuamente excluyentes y dicotómicas. $Y$ es que, como toda sociedad, los Andes peruanos no son solo tradicionales o modernos, sino, más bien, tradicionales y modernos, al mismo tiempo; con distintas tradiciones y modernidades en una misma área geográfica; y/o con tradiciones asentadas que, en algún punto histórico, fueron modernidades incorporadas.

De esta manera, en vez de utilizar las categorías de tradición y modernidad, convenimos en que es mejor utilizar las categorías de continuidades y cambios. Porque esta variación terminológica no solo implica un paso formal de unas categorías a otras, sino un proceso de descolonización de las mismas categorías de tradición y modernidad, que no hacen otra cosa que reproducir, en el fondo, la ya vieja dicotomía entre un ellos, los salvajes, y un nosotros, los civilizados (Comaroff y Comaroff, 1993). Por el contrario, hacer referencia a las continuidades (del pasado distante) no implica hablar de algo eterno $u$ originario, y hacer referencia a los cambios (del pasado reciente) no implica hablar únicamente de occidentalización, urbanización o globalización. Así, y de acuerdo con Hobsbawn (1983), las continuidades se construyen para establecer una estructura con respecto a un pasado histórico adecuado mientras la 
misma se encuentra inmersa en constantes innovaciones, negociaciones $\mathrm{y}$ redefiniciones de cambios que traen consigo las diferentes formas de relación con otras sociedades. Hablar de continuidades y cambios es, por eso, reconocer que ambas categorías se encuentran vinculadas entre sí, tanto que no se pueda pensar sobre las continuidades sin pensar sobre los cambios, y viceversa (Appadurai, 1996).

\section{Hacia una antropología digital: los fundamentos teóricos}

Este apartado explora, en primer lugar, los orígenes de la antropología digital desde una economía política de los medios de comunicación masiva y un enfoque poscolonial de las nuevas TIC. En segundo lugar, explora las teorías sobre la tecnología digital como práctica social desde los discursos (las microrrepresentaciones ideológicas conectadas a relaciones de poder) y los usos (las condiciones de domesticación productiva, económica y técnica de las nuevas TIC, y el rol de los contextos, actores, propósitos y resultados).

\subsection{Orígenes de la antropología digital}

Esta sección explora los orígenes de la antropología digital desde una economía política de los medios de comunicación masiva y desde un enfoque poscolonial de las nuevas TIC.

\subsubsection{Una economía política de los medios de comunicación masiva}

Desde finales del siglo XIX hasta la mitad del siglo $\mathrm{xx}$, influenciados por una perspectiva marxista sobre el acceso y la propiedad de los medios de producción, los teóricos clásicos de la comunicación de masas (Le Bon, 2000; Lippmann, 2003; Lasswell, 1948; Lazarsfeld, 1948) y los académicos de la Escuela de Frankfurt (Marcuse, 1954; Adorno y Horkheimer, 1972) coincidían en que los medios de comunicación masiva (prensa, radio y televisión) se pueden convertir en aparatos de reproducción ideológica, a partir de los cuales el poder hegemónico manipula el contenido cultural de la comunicación masiva, moldeando así formas de pensamiento y acción con el fin de mantener un sistema de dominación política y socioeconómica. Esta perspectiva se enfocó en los productores de los contenidos de los medios, así como en las mismas condiciones que rodean y estructuran la producción de la comunicación masiva, despojando de la posibilidad de toda agencia a los individuos, pues se asumía que las masas eran pasivas, homogéneas, manipulables, acríticas, socialmente aisladas y dependientes de la voluntad dominante de los productores.

Desde mediados del siglo $\mathrm{xx}$ se fue gestando un giro en los estudios sobre los medios de comunicación masiva, desde la perspectiva del consumidor, y con énfasis en la sociedad como audiencias activas, heterogéneas 
y socialmente relacionadas (Hovland, 1949; Festinger, 1957; Bauer, 1964). Esta perspectiva se enfocó en estudiar a los medios ya no como si fueran divinidades autoritarias omnipotentes, sino, más bien, como campos de interlocución de tribus mediáticas segmentadas, prosumidoras, negociantes y globalizadas (McQuail, 1972; Hall, 1973; Maffesoli, 1988; Jenkins, 1992; Thompson, 1995; Morley, 1996). Por lo tanto, el enfoque de estudio ya no se centraba en la producción, sino en los consumidores como individuos que resisten activamente los intentos de manipulación mediática, negociando u oponiéndose a los contenidos hegemónicos. De esta manera, desde este nuevo giro, se dejó de preguntar qué hacen los medios con los individuos para comenzar a cuestionarse qué es lo que hacen los individuos con los medios.

\subsubsection{Un enfoque poscolonial de las nuevas TIC}

Desde un enfoque poscolonial, existen considerables diferencias, hegemónicas y contrahegemónicas, entre los medios de comunicación masiva (los mass media) y las nuevas TIC (los new media). Y es que la propiedad y el acceso a los medios de comunicación masiva (prensa, radio y televisión) es un privilegio de una minoría hegemónica que concentra endogámicamente el poder de producir, colectivamente, verdades legitimadas, $y$, con ello, la posibilidad de manipular a la sociedad.
Pero, con el advenimiento y la democratización de las nuevas TIC (cámara fotográfica, filmadora, celular e internet) se generaron nuevas condiciones de producción, no solo porque, a partir del abaratamiento de los precios, se descentró exogámicamente a las masas la propiedad y/o el acceso a nuevos medios de producción, sino también porque permitió el empoderamiento contrahegemónico de una mayoría subalterna con la posibilidad de consumir, cuestionar y producir verdades tanto ajenas como propias. Ello, por supuesto, no implica que no existan discursos ideológicos y relaciones de poder dentro de los escenarios e interacciones con las nuevas TIC; ello sí implica que existe una mayor posibilidad de propiedad y acceso a medios de producción, $y$, por ende, a mejores relaciones de producción y a menores relaciones de dependencia y dominación.

Precisamente por ello, la antropología digital centra su atención en las personas en su relación con las tecnologías, y no en las tecnologías en sí mismas (Miller, 2000; Miller y Horst, 2012; Bargh y McKenna, 2004). Hablar de antropología digital implica estudiar los cambios culturales y estrategias adaptativas que se producen a partir de las interacciones sociales con las nuevas TIC (Porter, 1997) que han sido desarrolladas por el código binario de bits de 0s y 1s (Miller y Horst, 2012), tales como el celular y el internet, a diferencia de aquellos medios de comunicación, tales como la radio, el 
teléfono y la televisión, de corte análogo. En ese sentido, la antropología digital parte del objetivo de investigación de la cibernética para estudiar las relaciones entre los seres humanos y las máquinas, así como con las transformaciones sociales producto de estas relaciones (Wiener, 1988). En otras palabras, la antropología digital se interesa por estudiar los discursos y usos sociales de la tecnología y de los cambios culturales, a partir de la relación entre el mundo virtual de lo on line y el mundo material de lo off line (Boellstorff, 2012). Por ello, no es lo mismo hablar de antropología digital que de antropología virtual, porque esta última limita su campo de estudio a las ciberculturas (Lévy, 1997) y a las comunidades virtuales, con sus códigos de comunicación propios y reglas sociales específicas en el ciberespacio (Castells, 2000; Hine, 2000, 2012 y 2013; y Whitehead y Wesch, 2012). No obstante, no se debe analizar lo virtual y lo material como dos mundos distintos y separados uno del otro, sino, más bien, como una unidad dialéctica interrelacionada (Coleman, 2010). Por eso, si aceptamos, con la semiología, que un fenómeno existe y que es real desde que significa para individuos y colectivos, más allá de su manifestación material, audiovisual, digital o virtual, entonces, conviene hablar de vida material en vez de vida real, ya que esta última categoría en oposición a la de vida audiovisual, vida digital o vida virtual genera una errónea dicotomía entre realidad "verdadera" y realidad "falsa" (Rogers, 2009; Lehdonvirta, 2010).

\subsection{Debates sobre la tecnología oral y escrita}

Esta sección explora los debates sobre la tecnología oral y escrita desde las premisas de la Gran División, el Continuum Lingüístico y los Nuevos Estudios de Literacidad.

\subsubsection{La Gran División}

Las premisas de La Gran División señalan no solo que las tecnologías comunicativas oral y escrita son los instrumentos determinantes de una mentalidad, de una consciencia o de un modo de pensamiento y acción, sino también sostienen que hay diferencias exclusivas entre ambas tecnologías comunicativas.

Por eso, y cuestionando los postulados de Lord (1960), Parry (1971), Goody (1968), Ong (1982) y Havelock (1996), se vuelve necesario preguntar, en primer lugar, ¿las tecnologías comunicativas oral y escrita tienen inherentes e intrínsecas consecuencias cognitivas y sociales, posibilitando, así, el surgimiento de la cultura oral (metonímica, sintagmática, coordinada y argumentativa) y la cultura escrita (metafórica, paradigmática, subordinada y demostrativa? Y, en segundo lugar, ¿solo la escritura alfabético-fonética desarrolla las capacidades de categorización segmentada, abstracción descontextualizada, linealidad 
formal, razonamiento lógico y crítica analítica, entre otros procesos intelectuales; y solo la oralidad desarrolla las capacidades de acumulación repetitiva, narración rítmica, empatía emocional, despliegue sensorial e integración situacional?

Las evidencias psicológicas, antropológicas y sociolingüísticas en las que se basan tanto el Continuum Lingüístico como los Nuevos Estudios de Literacidad permiten señalar que los postulados de la Gran División no solo no recogen datos empíricos de trabajos de campo que prueben sus hipótesis, sino que también obvian evidencias etnológicas para caer en generalizaciones esencialistas. En ese sentido, para responder a las preguntas formuladas anteriormente, ni lo oral ni lo escrito generan de forma autónoma cambios cognitivos y sociales, y tampoco las habilidades atribuidas a la adquisición de la oral y lo escrito son exclusivas de cada una de estas tecnologías. De esta manera, las críticas a las premisas de la Gran División han permitido indicar que no se puede conceptualizar lo oral y lo escrito desde A). Una jerarquía evolucionista (mentalidad primitiva oral $<$ mentalidad civilizada escrita), pues es inaceptable desestimar sociedades humanas, pasadas y presentes, que producen, se comunican y funcionan oralmente, atribuyéndoles rótulos de primitivismo y salvajismo, a partir de su condición cultural diferente, y no menor, al de una civilización alfabetizada. Y B). Una oposición dicotómica (oralidad vs. escritura), porque oralidad y escritura no son categorías opuestas ni manifestaciones excluyentes, pues son presencias interrelacionadas; $\mathrm{ni}$, mucho menos, presentan un efecto universal, pues el impacto de lo oral y lo escrito no es igual en todos los tiempos y espacios, ni es culturalmente aislado ni socialmente descontextualizado.

\subsubsection{El Continuum Lingüistico}

Aunque no constituye una crítica radical al modelo propuesto por la Gran División, las premisas del Continuum Lingüístico cuestionan, a través de investigaciones cualitativas, el binario Oralidad-Escritura, postulando un acercamiento entre lo oral y lo escrito a modo de puente discursivo donde existen y coexisten, en usos contextualizados, tanto discursos orales informales (conversaciones casuales) y formales (exposiciones) como discursos escritos informales (cartas personales) y formales (artículos académicos).

Por un lado, Chafe (1982) ha encontrado que el lenguaje ritual séneca se asemejaba al lenguaje escrito, evidenciando, así, que es posible encontrar características atribuidas a lo escrito (como la formalidad, la linealidad y la subordinación) en contextos orales formales. Por otro lado, Tannen (1982) analizó personas letradas que utilizaban estrategias orales, y viceversa. Ambos pudieron constatar que lo escrito no reemplaza a la oral, y que existe un continuum entre sus usos contextuales, una superposición en el uso de ambas estrategias en varios escenarios. 


\subsubsection{Los Nuevos Estudios de Literacidad}

Si las premisas del Continuum Lingüístico critican el binario Oralidad-Escritura y señalan que lo oral y lo escrito no son recursos suplementarios, sino estrategias complementarias usadas contextualmente, los postulados de los Nuevos Estudios de Literacidad resaltarán los discursos ideológicos y las relaciones de poder dentro de los cuales se encuentran inmersas todas las literacidades (discursos y usos sociales situados de lectura y escritura), permitiendo así comprender cómo una literacidad oficial dominante (reseñas, artículos y libros, entre otras publicaciones legitimadas de la escolaridad, la academia y la tradición letrada occidental) no solo se posiciona por encima de una literacidad vernácula local (listas de compra, diarios íntimos, blogs y chats), sino que además usurpa otras prácticas sociales de lectura y escritura (Vich y Zavala, 2004; Zavala, NiñoMurcia y Ames, 2004).

Desde el acercamiento psicológico, Scribner y Cole (1981), después de realizar pruebas para examinar habilidades cognitivas tanto en sujetos que escribían en vai (que se enseña en los hogares y no en la escuela) como en sujetos que habían pasado por un proceso de escolarización oficial, concluyeron que solo en estos últimos (que habían pasado por un proceso de instrucción formal) se evidenciaban las habilidades cognitivas atribuidas a la escritura (capacidad metalingüística, pensamiento abstracto y categorización taxonómica, entre otras). Por su parte,
Bennet y Berry (1991) estudiaron, en el norte de Canadá, cómo el cri, un sistema de escritura prefabricado e introducido por un misionero, se propagó (alfabetizando) entre una población oral que hasta ese entonces carecía de escritura; y cómo los cri canadienses adoptaron las prácticas de escribir cartas, enviar notas, redactar crónicas y diarios personales así como llevar registro de asuntos familiares y comerciales, mientras su modo de expresión primordial siguió siendo la palabra hablada, y la función comunicativa de la cultura escrita silábica cri competía con la del teléfono, la radio y la televisión dentro de un medio ambiente lleno de antenas satelitales y chozas indias donde se ahúman pescados y carnes.

Desde los acercamientos antropológico y sociolingüístico, Street (2000), Akinnaso (1992), Heath (1982), Barton (1994), Barton y Hamilton (1998) enfatizaron la manera en que la lectoescritura es utilizada en prácticas sociales constituidas por contextos estructurados (como el hogar, el trabajo, la iglesia, la escuela) con patrones recurrentes dentro de los cuales se desarrollan e interiorizan repertorios orales y escritos (como normas, valores, actitudes, sentimientos, entre otras habilidades cognitivas) para ser usada en específicos contextos sociales.

\subsection{Teorías sobre la tecnología digital como práctica social}

Esta sección explora las teorías sobre la tecnología digital como práctica 
social desde los discursos (las microrrepresentaciones ideológicas conectadas a relaciones de poder) y los usos (las condiciones de domesticación productiva, económica y técnica de las nuevas TIC, y el rol de los contextos, actores, propósitos y resultados).

\subsubsection{Discursos: microrrepresentaciones ideológicas y relaciones de poder}

Nuestra línea de investigación se delimitó por los discursos y los usos como elementos fundamentales de la práctica social, y, por ello, como categorías centrales de estudio.

En primer lugar, los discursos son microrrepresentaciones ideológicas (posicionamientos, valoraciones y expectativas) que los sujetos construyen de sí mismos, de los otros y del mundo, en lo dicho y lo hecho, a través de distintas formas de expresión (orales, escritas, audiovisuales, digitales, virtuales, etc.) con el fin de negociar sus posiciones de poder, ya sea como dominantes, resistentes o dominados, y alcanzar intereses concretos dentro de interacciones específicas con los participantes y escenarios de un determinado contexto social de la vida cotidiana (Van Dijk, 1999 y 2005; Alvesson y Kärreman, 2000). Y si bien es cierto que existen también macrorrepresentaciones ideológicas, estas se encuentran, más bien, vinculadas a la producción y reproducción discursiva desde aparatos e instituciones hegemónicas, mientras que las microrrepresentaciones están relacionadas con prácticas sociales situadas.
Los discursos son un aspecto integral de la vida social, son vehículos primarios para la interacción de los individuos en sociedad, son facilitadores de producción y reproducción de lógicas colectivas de pensamiento y acción. Analizando los discursos accedemos a categorías, realidades, mentalidades e imaginarios colectivos, porque cuando decimos y hacemos cosas no solo transmitimos información, sino también comunicamos posiciones $\mathrm{y}$ representaciones sobre nosotros, sobre los otros y sobre el mundo (Shi-Xu, 2005). Por ello es que los discursos, en la interacción con el otro, no reflejan lo que somos, pensamos y sentimos sobre alguien o algo, sino más bien nuestro posicionamiento sobre alguien o algo (Burr, 1995); o, en otras palabras, los discursos no son neutrales, sino, más bien, son lenguajes situados de la interacción social que se despliegan contextualmente desde una perspectiva particular (Gee, 1992 y 1999) y desde un marco hermenéutico envestido por ideologías: representaciones que sustentan formas de dominio, versiones de la realidad conectadas a relaciones de poder (Edley, 2001).

Por otro lado, los usos son apropiaciones y utilizaciones de lo tecnológico en el desarrollo de las diversas dimensiones de la vida social cotidiana (Horst, 2012). De esta manera, los usos de las nuevas TIC, como veremos a continuación, deben ser comprendidos desde las condiciones de domesticación productiva, económica y técnica, pero también desde el 
rol de los contextos, actores, propósitos y resultados.

\subsubsection{Usos (I): domesticación productiva, económica y técnica}

Lo tecnológico se encuentra indisolublemente ligado a lo social. Por eso es fundamental el concepto de sistema sociotécnico (Pfaffenberger, 1992) no solo para enfatizar la socialidad de la actividad tecnológica humana, sino para definir lo tecnológico: ya no como técnicas y artefactos, y sí como una actividad que va de la cultura material (recursos, herramientas, habilidades y otros conocimientos) a la coordinación social de la producción.

Así, al importar menos el supuesto carácter intrínseco determinante de la tecnología, y sí más sus prácticas sociales en relación con contextos, actores, propósitos y resultados, el impacto del internet y el celular, en tanto tecnologías digitales, debe ser analizado, y siguiendo a Hahn y Kibora (2008), a partir de tres condiciones que son fundamentales para su uso: su domesticación productiva (si las nuevas TIC permiten impulsar el desarrollo de las actividades productivas de base $y / 0$ permiten la inserción en nuevas actividades productivas), su domesticación económica (si se poseen los recursos necesarios para adquirir y mantener las nuevas TIC) y su domesticación técnica (si se han adquirido los conocimientos necesarios para obtener el máximo provecho de las nuevas TIC), comprendiendo la domesticación como el proceso de aceptación y normalización de algo en la cotidianeidad de las diversas dimensiones de la vida social (Horst, 2012).

\subsubsection{Usos (II): contextos, actores, propósitos y resultados}

Las computadoras y los celulares no son solo máquinas pensantes, sino también máquinas sociales, pues funcionan como medios de comunicación e información y como medios de socialización, cuyas prácticas transforman nuestra vida material (Donath, 2014). Así, es necesario admitir que se debe estudiar las tecnologías siempre en relación con nuestro uso de las mismas, en sus relaciones entre ellas y en relación a un contexto específico dentro del cual son usadas (O'Donnell y Henriksen, 2002). En ese sentido, lo mismo que Olson, Hildyard y Torrance (1985) señalaban con respecto a la tecnología de la palabra escrita es aplicable a la tecnología digital: sus efectos en el cambio sociocultural no son directos, pues lo que importa no es lo que la tecnología le hace a la gente, sino lo que la gente hace con la tecnología en específicos contextos sociales (Foster, 1980; Street, 2000).

$\mathrm{Y}$ es que el proceso de inserción de las tecnologías no es automático ni se explica por una determinación tecnológica en sí misma, sino, más bien, por sus usos y sus apropiaciones materiales y simbólicas que dotan de sentido al aparato social y cultural con propósitos concretos (Larghi 
et al., 2012). Es por ello que Calcina (2013) ha recalcado la importancia de no asumir la tecnología como un fin en sí mismo, para no deificar la tecnología y no caer en un paradogma digital de determinismo tecnológico, porque las nuevas TIC (como toda tecnología) no tienen, en sí mismas, funciones de crecimiento, competitividad y desarrollo sostenible. Es por eso que es necesario concebir la tecnología digital como una práctica social: la tecnología digital situada dentro de un particular contexto cultural, por concretos actores, en específicos espacios y tiempos sociales, y con objetivos y resultados diferentes según las conductas, valoraciones y expectativas que se construyan alrededor de la misma.

\section{Nuevas TIC en los Andes peruanos: un balance bibliográfico}

Este apartado sitúa los tres rumbos actuales de los estudios dentro de la bibliografía sobre las transformaciones sociales y culturales vinculadas a las nuevas TIC en los Andes peruanos contemporáneos: en la expresión identitaria, en el desarrollo productivo y en la educación formal.

\subsection{Nuevas TIC y expresión identitaria}

Estas investigaciones enfatizan los usos de las nuevas TIC en sociedades rurales no solo con fines instrumentales de comunicación, sino también con objetivos culturales de representación identitaria y revitalización política. De este modo, estas transformaciones sociales (en la comunicación, representación y revitalización) evidencian la dialéctica entre las continuidades de una cultura local y los cambios de una cultura digital, mientras enfatizan el importante papel que desempeña el uso de nuevas TIC para la expresión identitaria andina.

Es fundamental el estudio de Huber (2002) sobre el impacto social de internet en las comunidades de Huamanga (Ayacucho, Perú). Dentro del ciberespacio (término acuñado por Gibson, hacia 1984, para definir espacios de organización, relación e interacción social ausentes de dimensiones territoriales demarcadas físicamente), los jóvenes universitarios y estudiantes de secundaria de clase media ayacuchanos encontraban que podían acceder a cualquier tipo de información (desde el material para preparar sus clases y resolver sus tareas hasta imágenes pornográficas y juegos en línea) y comunicarse rápidamente con otros (desde revisar correos electrónicos y comunicarse con familiares migrantes en otras ciudades nacionales e internacionales hasta chatear con gente desconocida para entablar amistad y/o conseguir relaciones amorosas).

Asimismo, Quinteros (2011) estudió cómo la apropiación de la cámara de video por Flaviano Quispe (Juliaca) y Nilo Inga (Huancayo) para producir cine desde/sobre los Andes (llamado 
también cine andino, provincial o regional, etnocéntricamente desde Lima) ha permitido comunicar narrativas locales (historias, costumbres, paisajes, personajes y música), pero también sentido de pertenencia e identidad, a las audiencias andinas que se congregan durante las proyecciones audiovisuales en sus plazas regionales. Van Der Zalm (2011), asimismo, estudió las percepciones sobre la proyección de una película en quechua en la plaza central de la comunidad de Huanoquite (Paruro, Cusco), hallando no solo que las personas afirmaban aprender cómo gestionar mejor su mundo a partir del contacto audiovisual con otros mundos, sino también que las mismas encontraban similitudes entre la narrativa audiovisual vista y su propia realidad vivida, $y$, por ello, se identificaban fílmicamente con sus experiencias de existencia (como el alcoholismo, la religión, el racismo, la ecología, la justicia campesina o los robos, asaltos y asesinatos). $Y$ es que el cine regional se ha convertido un tópico que ha recibido mucha atención en los últimos años debido a que gran parte de este se desarrolla sobre la base de tecnologías digitales. Así, también, Castro (2016) señala que las tecnologías digitales caseras generan una estética de la distorsión en las películas de terror producidas en el sur andino, pues se adaptan los patrones básicos del género de terror a locales tradiciones orales, luchando, con ello, por la visibilidad de sus propias memorias.
Del mismo modo, vale mencionar también el estudio de Underberg y Zorn (2013) sobre cómo el internet ha permitido transmitir contenidos de la cultura andina a través de formas virtuales de expresión. Así, asesorados por el retablista quechuahablante ayacuchano Nicario Jiménez, los autores pudieron desarrollar dos páginas web (Folkvine.org y Peru Digital Project) que comunicaban tanto pensamientos cosmológicos andinos como reflexiones sobre los problemas sociales contemporáneos, tales como la identidad, la migración y las relaciones interétnicas.

Por otro lado, vale mencionar cómo las agrupaciones musicales autoidentificadas como andinas, por ejemplo, La Sarita, Indiogenes y Alborada, utilizan las redes sociales como Youtube.com para difundir su propuesta cultural a través de la música. Particularmente, llama la atención grupos como Uchpa (cuyo significado quechua es cenizas), el cual, a través del rock de los amplificadores, las distorsiones y las guitarras eléctricas y de los sonidos andinos del arpa, el violín y el waqrapuku, entre otros, realiza, mayormente en quechua, nuevas versiones de viejos huaynos. Pero también es interesante mencionar al grupo Liberato Ex (Quinta Rima), el cual, a través de un DJ (disc jockey), realiza canciones de rap en quechua, con fines tanto de representación identitaria como de revitalización política.

Finalmente, y aunque no son investigaciones circunscritas a los Andes peruanos, es importante mencionar 
que en la Amazonía también se están utilizando los medios para producir narrativas particulares con el fin de resignificar tradiciones, y recuperar una memoria e historias colectivas que han sido relegadas de las narrativas nacionales de la cultura dominante y que están en peligro de ser olvidadas incluso dentro de su mundo local. Es interesante señalar que, a diferencia de lo que ocurre en los Andes, en la Amazonía peruana los medios han sido usados como tecnologías de autorrepresentación no solo con fines de revitalización y preservación cultural, sino también como una forma de movilización política. Así, por un lado, Espinosa de Rivero (1998) reveló que los pueblos amazónicos shipibo, aguaruna y ashaninka utilizaban la radio, la televisión y el internet con objetivos de autorrepresentación identitaria y visibilidad política. Por otro lado, Correa (2005) estudió cómo los ashaninkas de la comunidad indígena Marankiari Bajo utilizan el internet como un medio de representación virtual para reconstruir su identidad comunal desde conocimientos culturales locales hasta procesos contemporáneos. Asimismo, vale rescatar cómo los miembros de la comunidad indígena Kukama-Kukamiria del distrito de Santa Clara (Loreto), a través de la pieza audiovisual Kumbarikira, realizada por la ONG alemana Create Your Voice y Radio Ucamara, usan formas musicales no-nativas (desde el sonido de la batería electrónica del reggaetón hasta el fraseo rítmico del rap), no solo para autopromocionar, en español y kukama, el sentido de pertenencia a su identidad nativa, sino que además, ubicándose dentro de un contexto histórico de discriminación étnica, expresan sus fines de revitalización política lingüístico-cultural.

\subsection{Nuevas TIC y desarrollo productivo}

Estas investigaciones ponen en evidencia el importante rol que desempeñan los usos de las nuevas TIC para el desarrollo andino en distintos aspectos sociales: en la coordinación, supervisión, asesoramiento y eficiencia de la dimensión agropecuaria; en la transparencia y eficacia de la dimensión institucional; pero también dentro de la transformación social en el control, independencia, privacidad y entretenimiento de la dimensión familiar.

Diversos estudios se han abocado a estudiar el uso de la telefonía móvil en los Andes. Ruiz de Alonso (2009) ha estudiado el impacto del celular en las comunidades de Coasa (Puno), Yanaoca (Cusco) y Huarmaca (Piura), $y$ han encontrado que esta TIC es valorada mucho más en los lugares donde existen carencias de transporte (precisamente, por el ahorro de tiempo y de recursos monetarios). Así, se identificó este impacto en cinco dimensiones de la vida social de estas comunidades. En primer lugar, el impacto en la actividad agrícola se ha producido en la mejor comunicación con clientes, el asesoramiento sobre el producto y el modo de producirlo; en la reducción de 
la cadena de intermediarios, el aumento del tamaño del mercado nacional e internacional y el conocimiento de precios; en el acceso a asesorías técnicas, las coordinaciones del transporte preciso de mercadería y la gestión de otros negocios. En segundo lugar, el impacto en la actividad ganadera ha permitido la supervisión al pastor en el campo y la resolución de situaciones de emergencia tales como el abigeato, la pérdida de ganado o los accidentes humanos; el contacto con veterinarios para resolver enfermedades ganaderas, asesoría de personal especializado en reproducción ganadera y acceso a capacitaciones brindadas por ONG; y la posibilidad de solicitar insumos y medicinas y coordinar los precios y el transporte del ganado. En tercer lugar, el impacto en los servicios municipales y programas sociales ha posibilitado agilizar los trámites administrativos y la gestión de las municipalidades, mejorar las coordinaciones, supervisiones y controles de los programas sociales que ya existen en la zona, como el Vaso de Leche. En cuarto lugar, el impacto en la salud se ha materializado en la rápida ubicación de los profesionales que se encuentran prestando servicio fuera de su centro de salud y el rápido contacto y consulta de los profesionales de la salud con especialistas en ciudades más grandes. Y, en quinto lugar, el impacto en la educación se ha manifestado en la mejora de la comunicación entre las instituciones alejadas, padres-profesores, profesores-alumnos, $\mathrm{y}$ entre profesores.
Asimismo, León (2010) ha estudiado los usos de celulares e internet que hacen los funcionarios de los municipios de las comunidades de Quinua y Vinchos (Huamanga, Ayacucho). En primer lugar, los usos con respecto a la gestión y el envío de información mediante sistemas electrónicos en línea (así, el control de fondos públicos por parte del Estado, evitando la corrupción; la convocatoria pública de proveedores promueve la competencia democrática, evitando el nepotismo; y la posibilidad de llevar la contabilidad con rapidez y orden. Sin embargo, existen dificultades con respecto al sistema de costos de materiales, diseñado desde una perspectiva urbana; con la lenta atención de instituciones administradoras; así como con problemas de conectividad a raíz de la interrupción de la disponibilidad del fluido eléctrico y las lluvias). En segundo lugar, con respecto a la búsqueda de información para la gestión municipal (las páginas web de entidades públicas son referencias normativas y legales, así como también lo es el buscador on line Google y la red de contactos físicos de amistad, no interinstitucionales). En tercer lugar, con respecto a la comunicación institucional a través de correos electrónicos personales. En este punto es interesante la relación entre la información digital y el documento físico oficial, así como el desarrollo tanto de interacciones sociales digitales como materiales, con el objetivo de mantener relaciones económico-políticas permanentes. En cuarto lugar, con respecto 
a la coordinación interna (la mayoría de funcionarios cuenta con celulares, y aunque haya falta de cobertura, esta es solucionada con la ubicación de pequeñas zonas donde se capta la señal móvil). Y, en quinto lugar, con respecto a la comunicación con la población (con preferencia por la comunicación oral para coordinar visitas a la comunidad o para informar a los pobladores sobre obras).

Así, también, Bustamante (2011) estudió el uso efectivo de los celulares -es decir, la capacidad para integrar con éxito las nuevas tecnologías de información y comunicación al logro de objetivos individuales y colectivos (Gurstein, 2003)- para la productividad entre pequeños agricultores dentro del Valle de Huaral de la costa peruana. Este estudio reveló que el impacto de los celulares no solo se debía al hecho de que eran de bajo costo, simple manejo, personalizables y transportables, sino, sobre todo, al indispensable capital social -o sea, la capacidad para acceder a los recursos de la comunidad (Huysman y Wulf, 2004)- como herramienta de participación en asociaciones de productores e influencia sobre otros agricultores, y como motivador para obtener información agrícola a través de los celulares, que luego será retroalimentada con fuentes locales. Vale recordar que ya Alberti y Cotler (1972) hablaban de una orientación favorable del mundo andino ante los cambios tecnológicos locales, aprovechados por varios sectores de la población para autopromover el ascenso colectivo en dimensiones productivas como la agricultura.

Del mismo modo, Aronés, Barrantes y León (2011) estudiaron el uso de los celulares en ferias de las comunidades de Asillo y Taraco (Puno), y concluyeron que el proceso de universalización del celular en estas comunidades ha llevado a que el mismo deje de ser un instrumento de distinción socioeconómica, generacional y de género, así como también símbolo de reconocimiento, prestigio, estatus e instrucción formal. De esta manera, sea por alquiler o posesión, los pobladores se concentraban en las ferias no solo para vender productos y comprar lo que no se producía, sino también para efectuar llamadas. Así, el uso social cotidiano de los celulares ha influido en el ámbito familiar (sobre la ubicación y el control de los miembros) y en el ámbito laboral-productivo (sobre la ampliación y la consolidación de redes comerciales).

Por su parte, los estudios de Agüero y Barreto (2012) sobre el acceso a celulares y a internet en las comunidades de Pedregal (Piura) y Andaray (Arequipa) revelaron que el celular es usado como herramienta de trabajo para coordinar con empleadores e intermediarios y para establecer contacto comunicativo permanente con parientes lejanos, mientras que el internet es usado como medio de entretenimiento para escuchar música, revisar el correo electrónico, acceder a las redes sociales y a la información escolar para realizar los deberes del colegio. De 
esta manera, si bien se mantienen las brechas de residencia, generación y pobreza, el nuevo perfil de las mujeres rurales jóvenes reduce las brechas de género al conseguir insertarse en dinámicas territoriales y aportar al desarrollo de sus comunidades desde recientes estrategias de sociabilidad, considerando, por lo demás, que muchos jóvenes rurales acceden por primera vez a internet, y aprenden a usarlo, en la escuela, durante la clase de informática y siempre y cuando un profesor esté disponible.

En otro artículo sobre el acceso a celulares y a internet en las mismas comunidades, Asencio, Barreto y García (2013) han enfatizado el hecho de que el internet es usado más por los hombres para obtener información (ya que las mujeres tienen mayor carga doméstica y, por ello, menos tiempo libre) en tanto que los celulares son usados más por mujeres para entablar comunicación, así como en el hecho de que, a diferencia de lo que sucede con los celulares, el nivel educativo sí influye en el uso de internet (es decir, a más años de estudio, más y mejor uso del internet). Pero también han puesto énfasis en resaltar que el valor de los celulares no solamente es de corte comunicativo, sino también social, pues ha permitido a las mujeres jóvenes rurales ganar independencia del control de sus padres $\mathrm{y}$ de sus parejas en tanto les permite un espacio de privacidad y contribuye a su autonomía mientras conserva redes sociales y pertenencia a un grupo, y consolida y gestiona sus relaciones íntimas. Por último, se ha puesto énfasis en la movilización a pie hacia otros pueblos para tener acceso al internet, así como en el incremento radical de usos lúdicos de esta TIC (que, aunque todavía es valorada positivamente y no vista como un agente corruptor, es relacionada, recelosamente, con la distracción, la pornografía y las redes de captación de menores).

Por su parte, Hopkins et al. (2013) estudiaron el efecto del establecimiento de cabinas de internet como parte de proyectos de crecimiento y desarrollo con inclusión social en distintas comunidades de las provincias de Caylloma y Condesuyos (Arequipa), Espinar (Cusco), General Sánchez Cero (Moquegua) y El Collao (Puno). A diferencia de las cabinas privadas, las cabinas públicas instaladas con el apoyo de proyectos son percibidas como un bien público, y no como un bien exclusivo de cierto sector social. En ese sentido, las búsquedas en internet se concentran tanto en contenidos educativos como en la comunicación por correos electrónicos y en la información sobre problemas agrícolas y pecuarios, como plagas y enfermedades. Así, en estas comunidades, el internet tuvo varios efectos positivos. En primer lugar, la demanda por los servicios de internet en el mundo rural es más grande y diversificada de lo que inicialmente se tenía pensado (la demanda de internet no está vinculada solo con la producción y el mercado, sino también al sector educación por padres de familia, profesores 
y estudiantes). En segundo lugar, el internet facilita la inclusión económica y social (brinda información y posibilidades de mejoras económicas, servicios gubernamentales, tendencias en el mercado, alianzas políticas intercomunales, y revaloración de la identidad cultural y el orgullo comunal). En tercer lugar, se ha vuelto clave el desarrollo de capacidades en TIC en las comunidades rurales (la capacitación en herramientas virtuales y el desarrollo de habilidades locales con miras a un mejor aprovechamiento de internet). En cuarto lugar, la necesidad de revisar y fortalecer el modelo de gestión de las cabinas de internet (la sostenibilidad técnica de computadoras operativas y de fuerte señal, la sostenibilidad económica de recursos para cubrir gastos operativos, y la sostenibilidad de la gestión y responsabilidad para la continuidad de la oferta del servicio).

\subsection{Nuevas TIC y educación oficial}

Estas investigaciones ponen en evidencia el importante rol que desempeñan los programas oficiales de las nuevas TIC para la educación rural, enfatizando que el éxito de las mismas se encuentra relacionado directamente no solo con problemas de conectividad y de financiamiento, sino, sobre todo, con la capacitación docente sobre las nuevas TIC, el contexto cultural particular donde se inserta el proyecto y la utilidad social del mismo para la comunidad.
Paralelamente a estos usos cotidianos, es necesario observar también los usos de las TIC a través de los programas oficiales de educación. Es precursor el estudio de Trinidad (2002) en un caserío del distrito de La Merced (Áncash) sobre la importancia de la televisión, por encima del rol de la escuela, en la reconfiguración de las identidades locales y en el conocimiento sobre otros lugares y personas en contextos globales. Pero es todavía más interesante la experiencia, iniciada a principios del año 2007, por el programa Una laptop por niño (también conocido como Un portátil por niño, denominación que proviene del programa de la fundación norteamericana One Laptop Per Child - OLPC, de Nicholas Negroponte) realizado por el Ministerio de Educación con el objetivo de llevar computadoras a los niños de bajos recursos económicos de las zonas rurales del país.

Hasta la actualidad, el programa ha distribuido 797352 laptops por centros educativos públicos de cada uno de los departamentos del país, dato rescatado por Ames (2014) acerca de la cifra oficial del Ministerio de Educación del Perú sobre la distribución nacional de las computadoras del programa Una laptop por niño, previamente compradas por el Ministerio de Educación a la fundación OLPC. Asimismo, Ames nos recuerda que el programa oficial Una laptop por niño fue antecedido por el Proyecto Huascarán (2001-2006), el mismo que implicaba el uso compartido de acceso a internet a través de 
laboratorios de computadoras PC en las escuelas. El programa se inició en la comunidad de Arahuay (Lima), elegida por su relativa proximidad a la capital y por contar con una antena para la conexión inalámbrica a internet.

No obstante, como ha sucedido en otras comunidades, esta política oficial fracasó. Y es que, tal y como ya Trinidad (2005) y Gutiérrez (2009) lo habían señalado al estudiar las nuevas tecnologías de información y comunicación en proyectos educativos estatales, el hecho de implementar nuevas TIC en un centro educativo se encuentra en estrecha relación de éxito con una respectiva capacitación docente, pero también en la importancia de incorporar los conocimientos culturales locales de la población a estos nuevos medios, como llegaría a señalar Calcina (2013), al estudiar los impactos del internet y las condiciones en las que llega (sobre los problemas de conectividad, costos, capacitación y retroalimentación con respecto a otras tecnologías) en materia de desarrollo en la Amazonía.

En esa línea, tanto Kraemer, Dedrick y Sharma (2009) como Warschauer y Ames (2010) han señalado que el programa falla porque ignora el contexto social de aprendizaje donde se implementan los medios digitales. Es la evidencia de esta ruptura la que llevó a Laura (2010) a señalar que uno de los factores por los cuales este proyecto falla es por la falta de capacitación técnica, pedagógica, metodológica y epistemológica a los docentes. Así, también, Olivera (2011) apuntó que el programa no ha funcionado por la falta de proyectos que lo articulen, por la resistencia al cambio y por no tomar en cuenta a los actores involucrados, como se muestra también en otro artículo de Villanueva y Olivera (2012) sobre dos escuelas primarias de la periferia de Lima, en las cuales profesores y alumnos, a pesar de que ya se encontraban familiarizados con el uso de las computadoras con internet, ni encontraron reflejadas sus experiencias ni encontraron cumplidas sus expectativas, porque los agentes políticos externos habían insertado las computadoras sin guías de uso, capacitaciones ni objetivos claros (Balarin, 2013).

Tal vez esto se deba a que la escuela en los Andes, de acuerdo con Zavala (2002), sigue siendo un dominio letrado donde lo oral y lo escrito son distantes entre sí, y donde los textos escolares se vuelven elementos cerrados que no predican sobre la realidad inmediata de los individuos. En otras palabras, porque el contenido de la propuesta escolar en los Andes sigue aún construyéndose en nostálgica referencia a un modelo de escuela occidental que, evidentemente, no solamente no corresponde con el modelo andino, sino que ya no existe más. $\mathrm{Al}$ respecto, es interesante notar, como lo demuestra Ames (2014), que las TIC en muchas escuelas del Perú sigan siendo usadas como una extensión del libro, el cuaderno y la pizarra, anulando la posibilidad de desarrollar en niños y jóvenes habilidades digitales más complejas. 
Por último, Ames (2014) ha consolidado los previos hallazgos con su investigación en escuelas urbanas y rurales de La Libertad, Ucayali y Puno, reafirmando la importancia fundamental de los contextos sociales específicos y de los actores sociales particulares en la incorporación de las TIC, pero descubriendo, a la par de las brechas económicas, generacionales y de género, que existía una brecha de acceso material restringido a las TIC en la escuela (limitado a un espacio, un tiempo, una actividad y un instructor determinado) en comparación con el acceso más libre, activo y simultáneo que tienen los niños y jóvenes fuera de la misma (las cabinas, celulares y computadoras de casa), y para quienes las computadoras del programa de la fundación OLPC, llamadas también $\mathrm{XO}$, constituyen las tecnologías menos atractivas e importantes en sus vidas cotidianas.

\section{A modo de reflexión final: digitalización, empoderamiento e inclusión}

Estos puntuales acercamientos académicos sobre las nuevas TIC en los Andes peruanos (en materia de expresión identitaria, desarrollo productivo y educación formal) abren el camino para comprender, en profundidad, el papel transformador que vienen teniendo, desde hace no más de dos décadas, las nuevas TIC en las prácticas colectivas de producción y reproducción cultural en la base de la organización, relación e interacción social andina peruana. Por ello, a pesar del escaso número de publicaciones sobre las nuevas TIC en los Andes peruanos, estas investigaciones ya sientan las bases para continuar realizando trabajos de campo que estudien las prácticas sociales (discursos y usos) de las nuevas TIC dentro de las comunidades andinas actuales, con el objetivo de contribuir a la bibliografía de una imperante antropología peruana que busca comprender el mundo andino contemporáneo como el resultado histórico de diversas y diferentes tradiciones y modernidades, tanto pasadas como presentes, que han innovado, negociado y redefinido su complejo panorama de hoy.

Actualmente, y más allá de las cuestiones ideológicas del alfabetismo en el Perú como práctica de progreso cultural en sí misma, como lo han señalado ya Ames (2002) y Zavala (2002), no solamente la lectoescritura constituye una herramienta indispensable para el desarrollo competente de relaciones sociales, económicas y políticas en varios contextos rurales, urbanos y globales, sino también las capacidades desarrolladas en materia de hardware y software digitales con fines de uso para distintos contextos de la interacción, relación y organización social.

Pero, para que los individuos aprovechen productivamente el internet y el celular en su vida social no solo se requiere que estos desarrollen capacidades digitales a través de la 
capacitación técnica (o lo que también ha sido llamado alfabetización digital), sino, como requisito, que los mismos cuenten con posibilidades objetivas de acceso (condiciones óptimas de calidad, diversas de cantidad y baratas de precio). Por ello, si a pesar de las actuales y deficientes condiciones de acceso, sobre todo en cuanto al internet se refiere, muchos pobladores de las comunidades andinas han logrado, a partir de las nuevas TIC, impulsar viejas y nuevas actividades productivas (como la agricultura, la ganadería y el turismo), unas condiciones óptimas, diversas y baratas de acceso, en conjunto con un desarrollo efectivo de capacitaciones técnicas, contribuiría al incremento del desarrollo económico de muchas comunidades andinas.

No obstante, es importante rescatar también que el acceso a la alfabetización es también fundamental para posibilitar un aprovechamiento de la digitalización. Recordemos que ya Asencio, Barreto y García (2013) encontraron en las comunidades de Pedregal (Piura) y Andaray (Arequipa) que el nivel educativo sí influye en el uso de internet (es decir, a más años de estudio, mejor uso de internet); es decir, quienes han tenido mayor y mejor acceso a la alfabetización de la escolaridad, han obtenido mayores y mejores capacidades digitales $\mathrm{y}$, por ello, han podido apropiarse eficazmente del internet y el celular para desarrollar e impulsar sus actividades productivas. De hecho, hacia el 2013, el Congreso de la República del Perú aprobó el proyecto de ley n. 2183 de la alfabetización digital, con el objetivo de reducir la brecha digital (las desiguales condiciones de acceso y capacidades de manejo a las nuevas TIC) en sectores significativos de la población como el medio rural. $\mathrm{Y}$ a pesar de que dicho proyecto de ley enfatiza más la capacitación en competencias técnicas digitales, y no tanto las condiciones de acceso, es ya rescatable su proposición y aprobación legal, pues sienta un precedente para pensar una política de digitalización.

Así, pues, una política de digitalización (el desarrollo de posibilidades de acceso a nuevas TIC y el desarrollo de capacidades para el aprovechamiento productivo de dichas nuevas TIC) se vuelve un programa tan importante como el de la alfabetización. Así, ya sea desde políticas estatales o desde inversiones privadas, de lo que se trata es de comenzar a digitalizar las comunidades andinas que se encuentren, actualmente, semidigitalizadas o bajo una digitalización precaria (tanto por las deficientes posibilidades de acceso a las nuevas TIC en materia de cantidad, calidad y costos, como por las deficientes capacidades técnicas para aprovechar productivamente dichas nuevas TIC). En ese sentido, pues, se tendría que comenzar a pensar ya no solamente en programas de alfabetización, sino también en programas de digitalización para las comunidades andinas que promuevan no solamente el desarrollo de las posibilidades de acceso a las nuevas TIC, sino también el 
desarrollo de las capacidades de aprovechamiento para esas nuevas TIC.

Comprendida así la digitalización, cabría preguntarse si acaso esta no constituye ya un factor importante dentro de un proceso de empoderamiento de los individuos sociales con equitativas oportunidades de acceso y aprovechamiento de las nuevas TIC. De hecho, ya Silvera (2005), Castro (2005), Srinivasan (2006), Ortoll y Collado (2007), y Travieso y Planella (2008) han sostenido que el desarrollo de posibilidades y capacidades en torno a las nuevas TIC ha logrado reducir brechas que crean barreras de exclusión social, tales como la pobreza y la discriminación; y ya Galperin, Mariscal y Barrantes (2014) han planteado que el acceso al internet de banda ancha (parte del desarrollo de posibilidades) y la inversión en capital humano (parte del desarrollo de capacidades) son elementos vitales para el crecimiento económico. En definitiva, todo indica que una política de digitalización en los Andes que considere cada uno de los componentes previamente señalados, brindaría más y mejores oportunidades a los miembros de las comunidades para impulsar, por un lado, el desarrollo de sus actividades productivas y, por ello, su crecimiento económico; $y$, por el otro, el desarrollo de sus expresiones identitarias $y$, por ello, su visibilidad política.

\section{Referencias}

Adorno, T. y Horkheimer, M. (1972). Dialectics of Enlightenment. Nueva York: Herder and Herder.

Agüero, A. y Barreto, M. (2012). El nuevo perfil de las mujeres rurales jóvenes en el Perú. Lima: Documentos de Trabajo del programa Nuevas Trenzas, IEP.

Akinnaso, N. (1992). Schooling, Language, and Knowledge in Literate and Nonliterate Societies. Comparative Studies in Society and History, 34, 68-109.

Alberti, G. y Cotler, C. (1972). Aspectos sociales de la educación rural en el Perú. Lima: Perú Problema 8. IEP.

Alvesson, M. y Kärreman, D. (2000). Varieties of discourse: On the study of organizations through discourse analysis. Human Relations, número 53(9), 1125-1149.

Ames, P. (2002). Para ser iguales, para ser diferentes. Educación, escritura y poder en el Perú. Lima: IEP.

Ames, P. (2014). Niños y adolescentes frente a las nuevas tecnologías: acceso y uso de las tecnologías educativas en las escuelas peruanas. Revista Peruana de Investigación Educativa, (6), 147-172.

Appadurai, A. (1966). Modernity at Large. Cultural Dimensions of Globalization. Minneapolis: University of Minnesota Press.

Aronés, M.; Barrantes, R. y León, L. (2011). Todos tienen celular. Uso, apropiación e impacto de la telefonía móvil 
en el área de influencia de dos ferias en Puno, Perú. Documento de Trabajo número 161. Lima: IEP.

Asencio, R.; Barreto, M. y García, A. (2013). Control y trasgresión. El uso, apropiación e impacto de las TIC por las mujeres rurales jóvenes en el Perú. Lima: Documentos de Trabajo del programa Nuevas Trenzas, IEP.

Balarín, M. (2013). Las políticas TIC en los sistemas educativos de América Latina: caso Perú. Buenos Aires: UNICEF.

Bargh, J. y McKenna, K. (2004). The Internet and Social Life. Annual Review of Psychology, (55), pp. 573590, Palo Alto, California.

Barton, D. y Hamilton, M. (1998). Local Literacies: Reading and Writing in One Community. Londres: Routledge.

Barton, D. (1994). Literacy: an Introduction to the Ecology of Written Language. Oxford: Blackwell.

Bauer, R. (1964). The Obstinate Audience: The Influence Process from the Point of View of Social Communication. American Psychologist, 19(5), 319-328.

Bennet, J. A. y Berry, John W. (1991). Cree Syllabic Literacy: Cultural Context and Psychological Consequences. Tilburg: Tilburg University Press.

Boellstorff, T. (2012). Rethinking Digital Anthropology. En Daniel Miller (editor), Digital Anthropology, pp. 37-60. Oxford: Berg.

Burr, V. (1995). Social Constructionism. Londres: Routledge.
Bustamante, M. del C. (1988). Tecnologías campesinas de los Andes. Lima: Horizonte.

Bustamante, R. (2011). Información para la agricultura y capital social. Uso de smartphones entre pequeños agricultores en la costa peruana. Actas de la $V$ Conferencia ACORN-REDECOM, Lima.

Calcina, L. (2013). TIC para la Amazonía: ¿conectando el desarrollo?. En Paz, A., Paz Montoya, M. y Asensio, R. (editores), Escalando innovaciones rurales, pp. 279-292. Lima: IEP.

Castells, M. (2000). The Information Age: Economy, Society and Culture, Oxford: Blackwel.

Castro, J. (2005). La alfabetización digital como factor de inclusión social. La experiencia de la Red Conecta. Recuperado de http://www.redalyc. org/articulo.oa?id=201021059004, consultado el 20 de junio de 2015.

Castro, R. (2016). Cuentos de la cripta: filmes de horror y crisis social en los Andes. Revista Chilena de Antropología Visual, número 27, pp. 01-22, Santiago de Chile. Recuperado de http://www.rchav. cl/2016_27_art01_castro.html

Centro para el Desarrollo del Campesino y del poblador UrbanoMarginal (CEDECUM). (1988). Tecnología aymara: Revaloración del saber campesino. Lima: Proyecto de Tecnologías Campesinas, Cepia.

Chafe, W. (1982). Integration and Involvement in Speaking, Writing, 
and Oral Literature. En Tannen, D. (editora), Spoken and Written Language: Exploring Orality and Literacy, pp. 35-53. Norwood: Ablex.

Coleman, G. (2010). Ethnographic Approaches to Digital Media. Annual Review of Anthropology, 39, 487-505. California: University of California Press.

Comaroff, John y Comaroff, J. (1993). Modernity and its Malcontents: Ritual and Power in Post-Colonial Africa. Chicago: University of Chicago Press.

Correa, N. (2005). Asháninka online: ¿nuevas tecnologías, nuevas identidades, nuevos liderazgos? Una aproximación antropológica a la relación de la comunidad indígena Asháninka Marankiari Bajo con las tecnologías de información y comunicación (Tesis de licenciatura, Departamento de Antropología). PUCP.

Donath, J. (2014). The Social Machine. Designs for Living. Boston: MIT Press.

Edley, N. (2001). Analyzing Masculinity: Interpretative Repertoires, Ideological Dilemmas and Subject Positions. En Wetherell, M., Taylor, S. y Yates, S. (Editores), Discourse as Data, pp. 189-228. Londres: Sage.

Espinosa de Rivero, Ó. (1998). Los pueblos indígenas de la Amazonía peruana y el uso político de los medios de comunicación. América Latina Hoy, (19), 91-100.

Festinger, L. (1957). A Theory of Cognitive Dissonance. California: Stanford University Press.
Festival del libro puneño II. (1987). Culturas y tecnologías altoandinas, Tomo VI. Lima: Universo.

Foster, G.(1980). Lasculturas tradicionales y los cambios técnicos. México: Fondo de Cultura Económica.

Galperin, H.; Mariscal, J. y Barrantes, R. (2014). The Internet and Poverty. Opening the Black Box. Lima: Diálogo Regional sobre Sociedad de la Información.

Gee, J. P. (1992). The Social Mind: Language, Ideology and Social Practice. Nueva York: Bergin \& Garvey.

Gee, J. P. (1999). An Introduction to Discourse Analysis: Theory and Method. Londres: Routledge.

Gibson, W. (1984). Neuromancer. Nueva York: Ace.

Goody, J. (1968). Literacy in Traditional Societies. Cambridge: Cambridge University Press.

Gurstein, M. (2003). Effective Use: a Community Information Strategy Beyond the Digital Divide. Recuperado de http://firstmonday.org/ article/view/1107/1027, consultado el 18 de mayo del 2015.

Gutiérrez, G. (2009). Uso de las computadoras portátiles XO en el desarrollo de los componente del área de Comunicación Integral en los alumnos del sexto grado de la I.E. $n .^{\circ} 30115 \mathrm{del}$ centro poblado Chucupata en Junín (tesis de licenciatura. Departamento de Educación). PUCP.

Hahn, P. y Kibora, L. (2008). The Domestication of the Mobile Phone: 
Oral Society and New ICT in Burkina Faso. The Journal of Modern African Studies, 46, 87-109.

Hall, S. (1973). Encoding and Decoding in the Television Discourse. Birmingham: Centre for Contem-porary Cultural Studies.

Havelock, E. (1966). La musa aprende a escribir. Reflexiones sobre oralidad y escritura desde la antigüedad hasta el presente. Barcelona: Paidós.

Heath, S. (1982). Protean Shapes in Literacy: Ever-shifting Oral and Literate Traditions. En Tannen, D. (editora), Spoken and Written Language: Exploring Orality and Literacy, pp. 93-117. Norwood: Ablex.

Hine, Ch. (2000). Virtual Ethnography. Londres: Sage.

Hine, Ch. (2012). The Internet. Understanding qualitative research. Oxford: Oxford University Press.

Hine, Ch. (2013). Virtual Research Methods. Londres: Sage.

Hobsbawn, E. (1983). The Invention of Tradition. Cambridge: Cambridge University Press.

Horst,H.(2012). New Media Technologies in Everyday Life. En Miller, D. (editor), Digital Anthropology, pp. 67-79. Oxford: Berg.

Hopkins, R. et al. (2013). Las cabinas de internet como un instrumento de desarrollo e inclusión de los pueblos alto andinos del sur del Perú. En Paz, A., Montoya, M. P. y Raúl, R. (editores), Escalando innovaciones rurales, pp. 259-278. Lima: IEP.
Hovland, C. (1949). Experiments on Mass Communication. Princeton: Princeton University Press.

Huber, L. (2000). Consumo, cultura e identidad en el mundo globalizado: estudios de caso en los Andes. Lima: IEP.

Huysman, M. y Wulf, V. (2004). Social Capital and Information Technology. Boston: MIT Press.

Jenkins, H. (1992). Textual Poachers: Television Fans $\mathcal{E}$ Participatory Culture. Nueva York: Routledge.

Kraemer, K.; Dedrick, J. y Sharma, P. (2009). One Laptop per Child: Vision Versus Reality. Communications of the ACM, 6 (52), 66-73.

Largui, S. et al. (2012). La apropiación del acceso a computadoras e internet por parte de jóvenes de sectores populares urbanos en la Argentina. En Proenza, F. (editor), Tecnología y cambio social. El impacto del acceso público a las computadoras e internet en Argentina, Chile y Perú, pp. 17-67. Lima: IDRC-CRDI e IEP.

Lasswell, H. (1948). The Analysis of Political Behavior: an Empirical Approach. Londres: Routledge.

Laura, C. (2010). Una laptop por niño en escuelas rurales del Perú: un análisis de las barreras y facilitadores. Recuperado de siep.org.pe/ archivos/up/116.doc.

Lazarsfeld, P. (1948). The People's Choice: How the Voter Makes up his Mind in a Presidential Campaign. Nueva York: Columbia University Press. 
Le Bon, G. (2000). Psicología de las masas. Madrid: Morata.

Lechtman, H. y Soldi, A.M. (editores). (1981). La tecnología en el mundo andino, Runakunap Kausaynin Kupaq Rurasqankunaku, Tomo I: Subsistencia y mesuración. México: UNAM.

Lehdonvirta, V. (2010). Virtual Worlds Don't Exist: Questioning the Dichotomous Approach in MMO Studies. Game Studies, 10 (1). Recuperado de http://gamestudies. org/1001/articles/lehdonvirta.

León, L. (2010). Implicancias del uso de las tecnologías de la información y comunicación en municipios rurales. Un estudio de caso en Ayacucho, Perú. Recuperado de http://www.upf.edu/amymahan/_ pdf/101105_ponencia_LEON.pdf, consultado el 20 de mayo de 2015.

Lévy, P. (1997). Cyberculture. Rapport au Conseil de l'Europe dans le cadre du projet 'Nouvelles technologie: coopération culturelle et communication'. París: Odile Jacob.

Lippmann, W. (2003). La opinión pública. Madrid: Cuadernos de Langre.

Lord, A. (1960). A Singer of Tales. Cambridge: Harvard University Press.

Maffesoli, M. (1988). Le temps des Tribus. Le déclin de l'individualisme dans les sociétés de masse. Paris: MéridiensKlincksieck.

Marcuse, H. (1954). One-dimensional man. Boston: Bacon Press.
Miller, D. y Horst, H. (editores). (2012). Digital Anthropology. Oxford: Berg.

Miller, D. (editor). (2000). The Internet: An Ethnographic Approach. Oxford: Berg.

Morley, D. (1966). Televisión, audiencias y estudios culturales. Madrid: Amorrortu.

O'Donnell, D. y Henriksen, L. (2002). Philosophical Foundations for a Critical Evaluation of the Social Impact of ICT. Journal of Information Technology, 17, (2), 89-99.

Olivera, P. (2011). Usos y percepciones de los niños sobre el programa Una laptop por niño (tesis de Licenciatura, Departamento de Comunicación para el Desarrollo). PUCP.

Olson, D.; Hildyard, A. y Torrence, N. (1985). Literacy, Language and Learning. The Nature and Consequences of Reading and Writing. Oxford: Cambridge University Press.

Ong, W. (1982). Oralidad y escritura: tecnologías de la palabra. México: Fondo de Cultura Económica.

Ortoll, E.y Collado, A. J. (2007). La alfabetización digital en los procesos de inclusión social. Barcelona: UOC.

Parry, A. (1971). The Making of Homeric Verse. Oxford: Clarendon Press.

Pfaffenberger, B. (1992). Social Anthropology of Technology. Annual Review of Anthropology, (21), 491-516.

Porter, D. (editor). (1997). Internet Culture, Nueva York: Routledge. 
Quinteros, A. (2011). Entretejidos de imágenes: encuentros, brechas y memorias latentes en el nuevo cine andino. En Cánepa. G. (editora). Imaginación visual y cultura en el Perú, pp. 413-426. Lima: PUCP.

Rengifo, G. et al. (editor). (1986). Tecnologías campesinas de los Andes del Sur. Centro de Canelo de NOS (Chile) y CEPIA (Perú).

Rogers, R. (2009). The End of the Virtual. Digital Methods. Amsterdam: Vossiuspers UvA.

Ruíz de Alonso, L. (2009). Impacto de la telefonía celular en zonas rurales pobres del Perú. Recuperado de http:// www.acorn-redecom.org/papers/ LilianaRuiz.doc.

Shi-Xu. (2005). A Cultural Approach to Discourse. Basingstoke: Palgrave Macmillan.

Scribner, S. y Cole, M. (1981). The Psychology of Literacy, Cambridge: Harvard University Press.

Silvera, C. (2005). La alfabetización digital: una herramienta para alcanzar el desarrollo y la equidad en los países de América Latina y el Caribe. Recuperado de http://bvs. sld.cu/revistas/aci/vol13_1_05/aci04 105.htm.

Srinivasan, R. (2006). Indigenous, Ethnic, and Cultural Articulations of Media. International Journal of Cultural Studies, 4(9), 497-518.

Street, B. (2000). Literacy Events and Literacy Practices: Theory and Practice in the New Literacy Studies. En Jones, K. y Martin-Jones, M. (editoras). Multilingual Literacies. Reading and Writing Different Worlds, pp. 17-29. Philadelphia: John Benjamin's Publishing Company.

Tannen, D. (editora). (1982). Spoken and Written Language: Exploring Orality and Literacy. Norwood: Ablex.

Thompson, J. (1995). The Media and Modernity: A Social Theory of the Media, California: Stanford University Press.

Travieso, J. L. y Planella, J. (2008). La alfabetización digital como factor de inclusión social: una mirada crítica. Recuperado de http://www. uoc.edu/uocpapers/6/dt/esp/travieso_planella.pdf.

Trinidad, R. (2005). Entre la ilusión y la realidad: las nuevas tecnologías en dos proyectos educativos del Estado. Lima: IEP.

Trinidad, R. (2002). ¿Qué aprenden los niños del campo con la televisión? Globalización, socialización y aprendizaje. Lima: IEP.

Underberg, N. y Zorn, E. (2013). Digital Ethnography. Anthropology, Narrative and New Media. Austin: University of Texas Press.

Van der Zalm, J. (2011). Películas andinas para pueblos andinos: cine al aire libre en las provincias de Cusco y Apurímac. En Cánepa, G. (editora). Imaginación visual y cultura en el Perú, pp. 427-443. Lima: PUCP. 
Van Dijk, T. (1999). El análisis crítico del discurso. Anthropos, (186), 23-36.

Van Dijk, T. (2005). Ideología y análisis del discurso. Utopía y Praxis Latinoamericana, (29), 9-36.

Vich, V. y Zavala, V. (2004). Oralidad y poder: herramientas metodológicas. Lima: Grupo Norma.

Villanueva, E. y Olivera, P. (2012). Barreras institucionales para el desarrollo de una innovación: evaluando la implementación de las computadoras $\mathrm{XO}-1$ en dos escuelas periurbanas del Perú. Information Technologies \& International Development, 8(4), 191-203.

Warschauer, M. y Ames, M. (2010). Can One Laptop per Child Save the World's Poor?. Journal of International Affairs, 64(1), 33-51.
Whitehead, N. y Wesch, M. (2012). Human no More: Digital Subjectivities, Unhuman Subjects, and the End of Anthropology. Boulder: University Press of Colorado.

Wiener, N. (1988). The Human Use of Human Beings: Cybernetics and Society. Boston: Da Capo Press.

Zavala, V. (2002). Desencuentros con la escritura. Escuela y comunidad en los andes peruanos. Lima: Red para el Desarrollo de las Ciencias Sociales en el Perú.

Zavala, V., Niño-Murcia, M. y Ames, P. (2004). Escritura y sociedad: nuevas perspectivas teóricas y etnográficas, Lima: Universidad del Pacífico. 
